\title{
Topological Insulators at Room Temperature
}

\author{
Haijun Zhang ${ }^{1}$, Chao-Xing $\mathrm{Liu}^{2}$, Xiao-Liang $\mathrm{Qi}^{3}$, Xi Dai ${ }^{1}$, \\ Zhong Fang ${ }^{1}$, Shou-Cheng Zhang ${ }^{3}$ \\ ${ }^{1}$ Beijing National Laboratory for Condensed Matter Physics, \\ and Institute of Physics, Chinese Academy of Sciences, Beijing 100190, China \\ ${ }^{2}$ Center for Advanced Study, Tsinghua University, \\ Beijing, 100084, China ${ }^{3}$ Department of Physics, \\ McCullough Building, Stanford University, Stanford, CA 94305-4045
}

(Dated: December 10, 2008)

\begin{abstract}
Topological insulators are new states of quantum matter with surface states protected by the time-reversal symmetry. In this work, we perform first-principle electronic structure calculations for $S b_{2} \mathrm{Te}_{3}, \mathrm{Sb}_{2} \mathrm{Se}_{3}, \mathrm{Bi}_{2} \mathrm{Te}_{3}$ and $\mathrm{Bi} i_{2} S e_{3}$ crystals. Our calculations predict that $S b_{2} \mathrm{Te}_{3}, \mathrm{Bi}_{2} \mathrm{Te_{3 }}$ and $\mathrm{Bi}_{2} \mathrm{Se}_{3}$ are topological insulators, while $\mathrm{Sb}_{2} \mathrm{Se}_{3}$ is not. In particular, $\mathrm{Bi} \mathrm{i}_{2} \mathrm{Se}_{3}$ has a topologically non-trivial energy gap of $0.3 \mathrm{eV}$, suitable for room temperature applications. We present a simple and unified continuum model which captures the salient topological features of this class of materials. These topological insulators have robust surface states consisting of a single Dirac cone at the $\Gamma$ point.

PACS numbers:
\end{abstract}

Recently, the subject of time reversal invariant topological insulators has attracted great attention in condensed matter physics $1,2,3,4,5,6,7,8,9,10$. Topological states of quantum matter are defined and described by the corresponding topological terms in quantum field theory. For example, the quantum Hall effect is described by the topological Chern-Simons term 11 . On the other hand, the electromagnetic response of three dimensional (3D) topological insulators are described by the topological $\theta$ term of the form $S_{\theta}=\frac{\theta}{2 \pi} \frac{\alpha}{2 \pi} \int d^{3} x d t \mathbf{E} \cdot \mathbf{B}$, where $\mathbf{E}$ and $\mathbf{B}$ are the conventional electromagnetic fields and $\alpha$ is the fine structure constant. For a periodic system, all physical quantities are invariant under the shift of the parameter $\theta$ by integer multiples of $2 \pi$. Therefore, all time reversal insulators, including strongly correlated and disordered ones, fall into two disconnected classes, described $^{9}$ either by $\theta=0$ or by $\theta=\pi$. Topological insulators are defined by $\theta=\pi$, and this term physically described the topological magneto-electric effect ${ }^{\underline{9}}$. Topological insulators have surface or edge states with an odd numbers of gapless Dirac points.

The 2D topological insulator displaying the quantum spin Hall (QSH) effect was first predicted for the $\mathrm{HgTe}$ quantum wells $\stackrel{4}{*}$. Recently, the edge state transport has been experimentally observed in this system ${ }^{5}$. The electronic states of the 2D HgTe quantum wells are well described by a $2+1$ dimensional Dirac equation where the mass term is continuously tunable by the thickness of the quantum well. Beyond a critical thickness, the Dirac mass term of the 2D quantum well changes sign from being positive to negative, and a pair of gapless helical edge states appear inside the bulk energy gap. This microscopic mechanism for obtaining topological insulators by inverting the bulk Dirac gap spectrum can also be generalized to other 2D and 3D systems. The guiding principle is to search for insulators where the conduction and the valence bands have the opposite parity, and a "band inversion" occurs when the strength of some parameter, say the spin-orbit coupling, is tuned. For systems with inversion symmetry, a method based on the parity eigenvalues of band states at time reversal invariant points can be applied ${ }^{6}$. Based on this analysis, the $B i_{x} S b_{1-x}$ alloy has been predicted to be a topological insulator for a small range of $x$, and recently, surface states with an odd number of crossings at the fermi energy has been observed in angle-resolved photo-emission spectroscopy (ARPES) experiments 10 .

Since $B i_{x} S b_{1-x}$ is an alloy with random substitutional disorder, its electronic structures and dispersion relations are only defined within the mean field, or the coherent potential approximation (CPA). Its surface states are also extremely complex, with as many as five or possibly more dispersion branches, which are not easily describable by simple theoretical models. Alloys also tend to have impurity bands inside the nominal bulk energy gap, which could overlap with the surface states. Given the importance of topological insulators as new states of quantum matter, it is important to search for material systems which are stoimetric crystals with well defined electronic structures, preferably with simple surface states, and describable by simple theoretical models. In this work we focus on layered, stoimetric crystals $\mathrm{Sb}_{2} \mathrm{Te}_{3}, \mathrm{Sb}_{2} \mathrm{Se}_{3}$, $\mathrm{Bi}_{2} \mathrm{Te}_{3}$ and $\mathrm{Bi}_{2} \mathrm{Se}_{3}$. Our theoretical calculations predict that $\mathrm{Sb}_{2} \mathrm{Te}_{3}, \mathrm{Bi}_{2} \mathrm{Te}_{3}$ and $B i_{2} \mathrm{Se}_{3}$ are topological insulators while $\mathrm{Sb}_{2} \mathrm{Se}_{3}$ is not. Most importantly, our theory predicts that $\mathrm{Bi}_{2} \mathrm{Se}_{3}$ has a topologically non-trivial energy gap of $0.3 \mathrm{eV}$, therefore, it is a topological insulator at room temperature. The topological surface states for these crystals are extremely simple, described by a single gapless Dirac cone at the $\mathbf{k}=0 \Gamma$ point. We also propose a simple and unified continuum model which capture the salient topological features of this class of materials. In this precise sense, this class of 3D topological insulators share the great simplicity of the $2 \mathrm{D}$ topological 

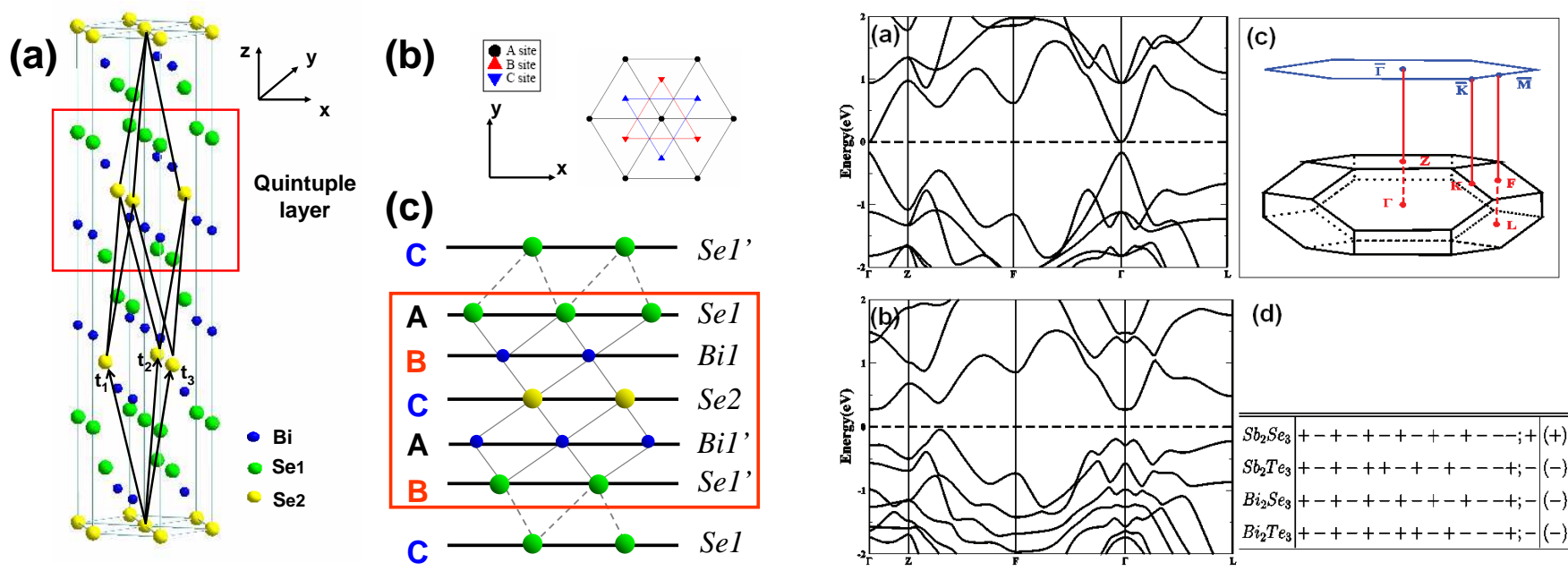

(d)

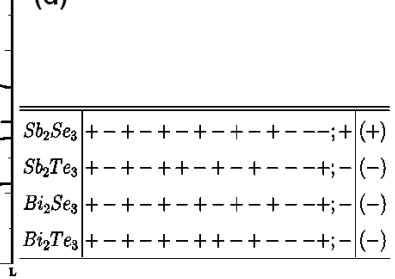

FIG. 2: band structure, Brillouin zone and parity eigenvalues. Band structure for $\mathrm{Bi}_{2} \mathrm{Se}_{3}$ without spin-orbit coupling(SOC) (a) and with SOC (b). The dashed line indicates Fermi level. (c) $\mathrm{BZ}$ for $\mathrm{Bi}_{2} \mathrm{Se}_{3}$ with space group $R \overline{3} \mathrm{~m}$. The four inequivalent time-reversal invariant points are $\Gamma(0,0,0), \mathrm{L}(\pi, 0,0), \mathrm{F}(\pi, \pi, 0)$ and $\mathrm{Z}(\pi, \pi, \pi)$. The blue hexagon shows the $2 \mathrm{D}$ BZ of projected $(1,1,1)$ surface, in which the high-symmetry $\mathbf{k}$ points $\bar{\Gamma}, \overline{\mathrm{K}}$ and $\overline{\mathrm{M}}$ are labeled. (d) The parity of the band at $\Gamma$ point for the four materials $\mathrm{Sb}_{2} \mathrm{Te}_{3}, \mathrm{Sb}_{2} \mathrm{Se}_{3}, \mathrm{Bi} \mathrm{i}_{2} \mathrm{Se}_{3}$ and $\mathrm{Bi} \mathrm{i}_{2} \mathrm{Te}$. Here we show the parities of fourteen occupied bands, including five $s$ bands and nine $\mathrm{p}$ bands, and the lowest unoccupied band. The product of the parities for the fourteen occupied bands is given in the bracket on the right of each row.

insulators realized in the $\mathrm{HgTe}$ quantum wells.

Band structure and parity analysis. $\mathrm{Bi}_{2} \mathrm{Se}_{3}, \mathrm{Bi}_{2} \mathrm{Te}_{3}$, $\mathrm{Sb}_{2} \mathrm{Te}_{3}$, and $\mathrm{Sb}_{2} \mathrm{Se}_{3}$ share the same rhombohedral crystal structure with the space group $D_{3 d}^{5}(R \overline{3} m)$ with five atoms in one unit cell. We take $B i_{2} S_{3}$ as an example and show its crystal structure in Fig. 1a, which has layered structures with triangle lattice within one layer. It has a trigonal axis (three fold rotation symmetry), defined as $\mathrm{z}$ axis, a binary axis (two fold rotation symmetry), defined as $\mathrm{x}$ axis, and a bisectrix axis (in the reflection plane), defined as y axis. The material consists of five-atom layers arranged along $z$ direction, known as quintuple layers. Each quintuple layer consists of five atoms with two equivalent $S e$ atoms (denoted as $S e 1$ and $S e 1^{\prime}$ in Fig. 1b), two equivalent $B i$ atoms (denoted as $B i 1$ and $B i 1^{\prime}$ in Fig. 1b), and a third $S e$ atom (denoted as $S e 2$ in Fig. 1b). The coupling is strong between two atomic layers within one quintuple layer but much weaker, predominantly of the van der Waals type, between two quintuple layers. The primitive lattice vectors $\mathbf{t}_{1,2,3}$ and rhombohedral unit cells are shown in Fig. 1(a). $S e 2$ site plays the role of inversion center and under inversion operation, $B i 1$ is changed to $B i 1^{\prime}$ and $S e 1$ is changed to $S e 1^{\prime}$. The existence of inversion symmetry enable us to construct eigenstates with definite parity for this system.

$A b$ initio calculations for $S b_{2} \mathrm{Te}_{3}, \mathrm{Sb}_{2} \mathrm{Se}_{3}, \mathrm{Bi}_{2} \mathrm{Te} e_{3}$ and
$\mathrm{Bi}_{2} \mathrm{Se}_{3}$ are carried out in the framework of PBE-type $\frac{12}{}$ generalized gradient approximation(GGA) of the density functional theory (DFT) $\stackrel{13,14}{\underline{1}}$. BSTATE package $\frac{15}{\underline{15}}$ with plane-wave pseudo-potential method is used with $\mathbf{k}$-point grid taken as $10 \times 10 \times 10$ and the kinetic energy cutoff fixed to $340 \mathrm{eV}$. For $\mathrm{Sb}_{2} \mathrm{Te}_{3}, B i_{2} \mathrm{Te} e_{3}$ and $B i_{2} \mathrm{Se}_{3}$, the lattice constants are chosen from experiments ${ }^{16}$, while for $\mathrm{Sb}_{2} \mathrm{Se}_{3}$, the lattice parameters are optimized in the selfconsistent calculation for rhombohedral crystal structure ( $a=4.076 \AA, c=29.830 \AA)$, due to the lack of experiment data.

Our results are consistent with the previous calculations 17,18 . In particular, we note that $B i_{2} S_{3}$ has an energy gap about $0.3 \mathrm{eV}$, which agrees well with the experimental data (about $0.2-0.3 \mathrm{eV}) \stackrel{19,20}{ }$. In the following, we take the band structure of $\mathrm{Bi}_{2} \mathrm{Se}_{3}$ as an example. Fig. 2 (a) and (b) show the band structure of $\mathrm{Bi}_{2} \mathrm{Se}_{3}$ without spin-orbit coupling (SOC) and with $\mathrm{SOC}$, respectively. By comparing the two figures one can see clearly that the only qualitative change induced by turning on SOC is an anti-crossing feature around $\Gamma$ point, which thus indicates an inversion between the conduction band and valence band due to SOC effect, suggesting $\mathrm{Bi}_{2} \mathrm{Se}_{3}$ to be a topological insulator. To firmly establish the topological nature of this material, we follow the method proposed by $\mathrm{Fu}$ and $\mathrm{Kane}^{\underline{\underline{6}}}$ and 
calculate the product of the parities of the Bloch wavefunction for the occupied bands at all the time-reversal invariant momenta $\Gamma, F, L, Z$ in Brillouin zone (BZ). As expected, we find that at $\Gamma$ point the parity of one occupied band is changed upon turning on SOC, while the parity remains unchanged for all occupied bands at other momenta $F, L, Z$. Since the system without SOC is guaranteed to be a trivial insulator, we conclude that $\mathrm{Bi}_{2} \mathrm{Se}_{3}$ is a strong topological insulator. The same calculation is performed for the other three materials, from which we find that $S b_{2} T e_{3}$ and $B i_{2} T e_{3}$ are also strong topological insulators, and $\mathrm{Sb}_{2} \mathrm{Se}_{3}$ is a trivial insulator. The parity eigenvalues of the highest 14 bands below the fermi level and the first conduction band at $\Gamma$ point are listed in Fig. $2(\mathrm{~d})$. From this table we can see that the product of parities of occupied bands at $\Gamma$ point changes from the trivial material $S b_{2} S e_{3}$ to the three non-trivial materials, due to an exchange of the highest occupied state and the lowest unoccupied state. This agrees with our earlier analysis that an inversion between the conduction band and valence band occurs at $\Gamma$ point.

To get a better understanding of the inversion and the parity exchange, we start from the atomic energy levels and consider the effect of crystal field splitting and spinorbit coupling to the energy eigenvalues at $\Gamma$ point, which is summarized schematically in three stages (I), (II) and (III) in Fig. 3 (a). Since the states near Fermi surface are mainly coming from $p$ orbitals, we will neglect the effect of $s$ orbitals and starting from the atomic $p$ orbitals of $B i\left(6 s^{2} 6 p^{3}\right)$ and $S e\left(4 s^{2} 4 p^{4}\right)$. In stage (I), we consider the chemical bonding between $B i$ and $S e$ atoms within a quintuple layer, which is the largest energy scale in the current problem. First we can recombine the orbitals in a single unit cell according to their parity, which results in three states (two odd one even) from each $S e p$ orbital and two states (one odd one even) from each Bi $p$ orbital. The formation of chemical bonding hybridize the states on $B i$ and $S e$ atoms, thus push down all the $S e$ states and lift up all the $B i$ states. In Fig. 3 (a), these five hybridized states are labeled as $\left|P 1_{x, y, z}^{ \pm}\right\rangle,\left|P 2_{x, y, z}^{ \pm}\right\rangle$and $\left|P 0_{x, y, z}^{-}\right\rangle$, where the superscripts,+- stand for the parity of the corresponding states. In stage (II), we consider the effect of the crystal field splitting between different $p$ orbitals. According to the point group symmetry, the $p_{z}$ orbital is split from $p_{x}$ and $p_{y}$ orbitals while the latter two remain degenerate. After this splitting, the energy levels closest to the Fermi energy turn out to be the $p_{z}$ levels $\left|P 1_{z}^{+}\right\rangle$and $\left|P 2_{z}^{-}\right\rangle$. In the last stage (III), we take into account the effect of SOC. The atomic SOC Hamiltonian is given by $H_{s o}=\lambda \vec{l} \cdot \vec{S}$, with $l, S$ the orbital and spin angular momentum, and $\lambda$ the SOC parameter. The SOC Hamiltonian mixes spin and orbital angular momenta while preserving the total angular momentum, which thus leads to a level repulsion between $\left|P 1_{z}^{+}, \uparrow\right\rangle$ and $\left|P 1_{x+i y}^{+}, \downarrow\right\rangle$, and similar combinations. Consequently, the $\left|P 1_{z}^{+}, \uparrow(\downarrow)\right\rangle$ state is pushed down by the SOC effect and the $\left|P 2_{z}^{-}, \uparrow(\downarrow)\right\rangle$ state is pushed up. If the SOC is large
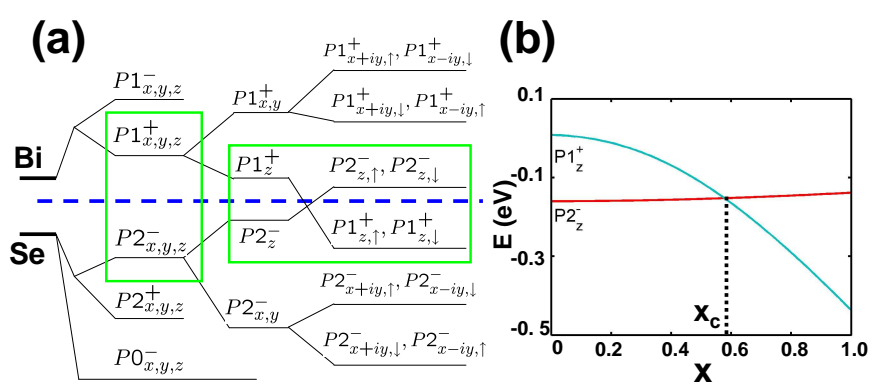

(I) (II) (III)

FIG. 3: Band Sequence. (a) Schematic picture of the evolution from the atomic $p_{x, y, z}$ orbitals of $B i$ and $S e$ into the conduction and valence bands of $\mathrm{Bi}_{2} \mathrm{Se}_{3}$ at $\Gamma$ point. The three different stages (I), (II) and (III) represent the effect of turning on chemical bonding, crystal field splitting and spin-orbit coupling, respectively (see text). The blue dashed line represents the Fermi energy. (b) The energy levels $\left|P 1_{z}^{+}\right\rangle$and $\left|P 2_{z}^{-}\right\rangle$of $B i_{2} S e_{3}$ at $\Gamma$ point versus an artificially rescaled atomic spin-orbit coupling $\lambda(B i)=x \lambda_{0}(B i)=$ $1.25 x \mathrm{eV}, \lambda(S e)=x \lambda_{0}(S e)=0.22 x \mathrm{eV}$ (see text). A level crossing occurs between these two states at $x=x_{c} \simeq 0.6$.

enough $\left(\lambda>\lambda_{c}\right)$, the order of these two levels is reversed. To see this inversion process explicitly, we also calculate the energy levels $\left|P 1_{z}^{+}\right\rangle$and $\left|P 2_{z}^{-}\right\rangle$for a model Hamiltonian of $\mathrm{Bi}_{2} \mathrm{Se}_{3}$ with artificially rescaled atomic SOC parameters $\lambda(B i)=x \lambda_{0}(B i), \lambda(S e)=x \lambda_{0}(S e)$, as shown in Fig. $3(\mathrm{~b})$. Here $\lambda_{0}(B i)=1.25 \mathrm{eV}$ and $\lambda_{0}(S e)=0.22 \mathrm{eV}$ are the realistic value of $B i$ and $S e$ atomic SOC parameters, respectively. ${ }^{21}$ From Fig. 3 (b) one can see clearly that a level crossing occurs between $\left|P 1_{z}^{+}\right\rangle$and $\left|P 2_{z}^{-}\right\rangle$ when the SOC is about $60 \%$ of the realistic value. Since these two levels have opposite parity, the inversion between them drives the system into a topological insulator phase. Therefore, the mechanism for the 3D topological insulator in this system is exactly analogous to the mechanism in the 2D topological insulator of $\mathrm{HgTe}$. In summary, through the analysis above we find that $\mathrm{Bi}_{2} \mathrm{Se}_{3}$ is topologically nontrivial due to the inversion between two $p_{z}$ orbitals with opposite parity at $\Gamma$ point. Similar analysis can be carried out on the other three materials, from which we see that $S b_{2} T e_{3}$ and $B i_{2} T e_{3}$ are qualitatively the same as $B i_{2} S e_{3}$, while the SOC of $S b_{2} T e_{3}$ is not strong enough to induce such an inversion.

Topological surface states. The existence of topological surface states is one of the most important properties of the topological insulators. To see the topological features of the four systems explicitly, we calculate the surface states of these four systems based on $a b$ initio calculation. First we construct the maximally localized Wanneir function(MLWF) from the $a b$ initio calculation using the method developed by N. Marzari et al.22,23. With these MLWF hoping parameters, we employ iterative $\operatorname{method}^{24,25}$ to obtain the surface Green function of 

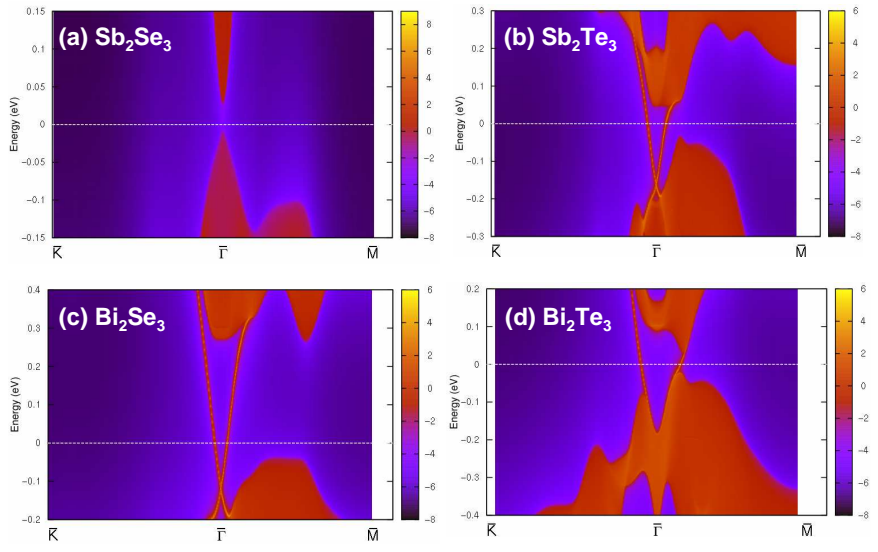

FIG. 4: Surface states. Energy and momentum dependence of the local density of states (LDOS) for (a) $\mathrm{Sb}_{2} \mathrm{Se}_{3}$, (b) $\mathrm{Sb}_{2} \mathrm{Te}_{3}$, (c) $\mathrm{Bi}_{2} \mathrm{Se}_{3}$ and (d) $B i_{2} \mathrm{Te} e_{3}$ on the [111] surface. Here warmer color represents higher LDOS. The red regions indicate bulk energy bands and the blue regions indicate bulk energy gap. The surface states can be clearly seen around $\Gamma$ point as red lines dispersing in the bulk gap for $S b_{2} T e_{3}$, $B i_{2} \mathrm{Se}_{3}$ and $B i_{2} \mathrm{Te}_{3}$. No surface state exists for $\mathrm{Sb}_{2} S e_{3}$.

the semi-infinite system. The imaginary part of the surface Green function is the local density of states (LDOS), from which we can obtain the dispersion of the surface states. When calculating the surface Green function, we only use the bulk's MLWF hoping parameters as semiinfinite systems without considering surface corrections. Due to the layered structure of these materials, we expect the surface reconstruction effect to be minor for [111] surface. The surface LDOS on the [111] surface for all the four systems are shown in Fig. 4. For $S_{2} \mathrm{Te}_{3}$, $B i_{2} \mathrm{Se}_{3}$ and $\mathrm{Bi}_{2} \mathrm{Te}_{3}$, one can clearly see the topological surface states which form a single Dirac cone at $\Gamma$ point. On comparison $\mathrm{Sb}_{2} \mathrm{Se}_{3}$ has no surface state and is a topological trivial insulator. Thus the surface state calculation agrees well with the bulk parity analysis, and confirm conclusively the topologically nontrivial nature of the three materials. For $\mathrm{Bi}_{2} \mathrm{Se}_{3}$ the Fermi velocity of the topological surface states is $v_{F} \simeq 5.0 \times 10^{5} \mathrm{~m} / \mathrm{s}$, which is similar to that of the other two materials.

Low energy effective model. Since the topological nature is determined by the physics near $\Gamma$ point, it is possible to write down an simple effective Hamiltonian to characterize the low-energy long-wavelength properties of the system. Starting from four low lying states $\left|P 1_{z}^{+}, \uparrow(\downarrow)\right\rangle$ and $\left|P 2_{z}^{-}, \uparrow(\downarrow)\right\rangle$ at $\Gamma$ point, such a Hamiltonian can be constructed by the theory of invariants 26 for the finite wavevector $\mathbf{k}$. Based on the symmetries of the system, the generic form of the $4 \times 4$ effective Hamiltonian can be written down up to the order of $O\left(\mathbf{k}^{2}\right)$, and the tunable parameters in the Hamiltonian can be obtained by fitting the band structure of our $a b$ initio calculation. The important symmetries of the system are time-reversal symmetry $T$, inversion symmetry $I$ and three fold rotation symmetry $C_{3}$ along the $z$ axis. In the basis of $\left(\left|P 1_{z}^{+}, \uparrow\right\rangle,\left|P 2_{z}^{-}, \uparrow\right\rangle,\left|P 1_{z}^{+}, \downarrow\right\rangle,\left|P 2_{z}^{-}, \downarrow\right\rangle\right)$, the representation of the symmetry operations are given by $T=$ $\mathcal{K} \cdot i \sigma^{y} \otimes \mathrm{I}_{2 \times 2}, I=\mathrm{I}_{2 \times 2} \otimes \tau_{3}$ and $C_{3}=\exp \left(i \frac{\pi}{3} \sigma^{z} \otimes \mathrm{I}_{2 \times 2}\right)$, where $\mathcal{K}$ is the complex conjugation operator, $\sigma^{x, y, z}$ and $\tau^{x, y, z}$ denote the Pauli matrices in the spin and orbital space, respectively. By requiring these three symmetries and keeping only the terms up to quadratic order in $\mathbf{k}$, we obtain the following generic form of the effective Hamiltonian:

$$
\begin{aligned}
& H(\mathbf{k})=\epsilon_{0}(\mathbf{k}) \mathrm{I}_{4 \times 4}+ \\
& \left(\begin{array}{cccc}
\mathcal{M}(\mathbf{k}) & A_{1} k_{z} & 0 & A_{2} k_{-} \\
A_{1} k_{z} & -\mathcal{M}(\mathbf{k}) & A_{2} k_{-} & 0 \\
0 & A_{2} k_{+} & \mathcal{M}(\mathbf{k}) & -A_{1} k_{z} \\
A_{2} k_{+} & 0 & -A_{1} k_{z} & -\mathcal{M}(\mathbf{k})
\end{array}\right)+o\left(\mathbf{k}^{2}\right)
\end{aligned}
$$

with $k_{ \pm}=k_{x} \pm i k_{y}, \epsilon_{0}(\mathbf{k})=C+D_{1} k_{z}^{2}+D_{2} k_{\perp}^{2}$ and $\mathcal{M}(\mathbf{k})=M-B_{1} k_{z}^{2}-B_{2} k_{\perp}^{2}$. By fitting the energy spectrum of the effective Hamiltonian with that of the $a b$ initio calculation, the parameters in the effective model can be determined. For $B i_{2} S e_{3}$, our fitting leads to $M=0.28 \mathrm{eV}, A_{1}=2.2 \mathrm{eV} \cdot \AA, A_{2}=4.1 \mathrm{eV} \cdot \AA, B_{1}=10 \mathrm{eV}$. $\AA^{2}, B_{2}=56.6 \mathrm{eV} \cdot \AA^{2}, C=-0.0068 \mathrm{eV}, D_{1}=1.3 \mathrm{eV} \cdot \AA^{2}$, $D_{2}=19.6 \mathrm{eV} \cdot \AA^{2}$. Except for the identity term $\epsilon_{0}(\mathbf{k})$, the Hamiltonian (1) is nothing but the 3D Dirac model with uniaxial anisotropy along $z$ direction and $\mathbf{k}$ dependent mass terms. From the fact $M, B_{1}, B_{2}>0$ we can see that the order of the bands $\left|T 1_{z}^{+}, \uparrow(\downarrow)\right\rangle$ and $\left|T 2_{z}^{-}, \uparrow(\downarrow)\right\rangle$ are inverted around $\mathbf{k}=0$ compared with large $\mathbf{k}$, which correctly characterizes the topologically non-trivial nature of the system. Such an effective Dirac model can be used for further theoretical study of the $\mathrm{Bi}_{2} \mathrm{Se}_{3}$ system, as long as the low energy properties are concerned. For example, as one of the most important low energy properties of the topological insulators, the topological surface states can be obtained from diagonalizing the effective Hamiltonian (1) with an open boundary condition, with the same method used in the study of two-dimensional quantum spin Hall insualtor ${ }^{27}$. For a surface perpendicular to the $z$ direction (i.e., [111] direction), the surface states are described by a $2 \times 2$ massless Dirac Hamiltonian

$$
H_{\text {surf }}\left(k_{x}, k_{y}\right)=\left(\begin{array}{cc}
0 & A_{2} k_{-} \\
A_{2} k_{+} & 0
\end{array}\right)
$$

in the basis of $(|\mathbf{k}, \uparrow\rangle,|\mathbf{k}, \downarrow\rangle)$. Here the surface state wavefunction $|\mathbf{k}, \uparrow(\downarrow)\rangle$ is a superposition of the $\left|P 1_{z}^{+}, \uparrow(\downarrow)\right\rangle$ and $\left|P 2_{z}^{+}, \uparrow(\downarrow)\right\rangle$, respectively. For $A_{2}=$ $4.1 \mathrm{eV} \cdot$ Aobtained from the fitting, the fermi velocity of surface states is given by $v_{F}=A_{2} / \hbar \simeq 6.2 \times 10^{5} \mathrm{~m} / \mathrm{s}$, which agrees reasonably with the $a b$ initio results shown in Fig. 4 (c). In summary, the surface effective theory (2) characterizes the key features of the topological surface states, and can be used in future to study the surface state properties of the $\mathrm{Bi}_{2} \mathrm{Se}_{3}$ family of topological insulators.

In conclusion we have theoretically predicted a new class of topological insulators $\mathrm{Sb}_{2} \mathrm{Te}_{3}, \mathrm{Bi}_{2} \mathrm{Te} \mathrm{e}_{3}$ and 
$\mathrm{Bi}_{2} \mathrm{Se}_{3}$. In particular, $\mathrm{Bi}_{2} \mathrm{Se}_{3}$ has a large topologically non-trivial energy gap $\sim 0.3 \mathrm{eV}$, sufficient for room temperature operation. The topologically nontrivial nature of these three materials originates from a band inversion at $\Gamma$ point, similar to the strained 3D $H g T e^{6.28}$ and twodimensional $\mathrm{HgTe}$ quantum wells ${ }^{4}$. The topologically robust surface states are studied by ab initio method, which consist of a single Dirac cone around the $\Gamma$ point. We have also obtained a $4 \times 4$ effective theory to characterize the bulk properties at low-energy and long-wavelength, and a $2 \times 2$ massless Dirac model to describe the surface states.

The topological surface states can be directly verified by various experimental techniques, such as ARPES and scanning tunneling microscopy (STM). In the recent years, evidences of surface states have been observed for $\mathrm{Bi}_{2} \mathrm{Se}_{3}$ and $\mathrm{Bi}_{2} \mathrm{Te}_{3}$ in ARPES ${ }^{29}$ and $\mathrm{STM}^{30}$ experiments. In particular, the surface states of $B i_{2} T e_{3}$ observed in Ref ${ }^{29}$ had a similar dispersion as we obtained in Fig. 4 (d), which were also shown to be quite stable and robust, regardless of photon exposure and temperature. Thus this experimental result strongly supports that the surface states have topological origin. Further experimental studies on the surface state properties, such as ARPES, STM and transport measurements are necessary to verify our prediction. Moreover, the 3D topological insulators are predicted to display the universal topological magneto-electric effect $\underline{\underline{9}}$ when the surface is coated with a thin magnetic film. Compared with the $B i_{1-x} S b_{x}$ alloy, the surface states of the $B i_{2} S e_{3}$ family of topological insulators contain only a single fermi pocket, making it easier to open up a gap on the surface by magnetization and to observe the topological Faraday/Kerr rotation ${ }^{\underline{9}}$ and image magnetic monopole effect ${ }^{31}$. If observed, such effects can be unambiguously identified as the experimental signature of the non-trivial topology of the electronic properties.
1 Day, C. Quantum spin Hall effect shows up in a quantum well insulator, just as predicted. Phys. Today 61, 19 (2008).

2 C. L. Kane \& E. J. Mele. Quantum spin Hall effect in graphene. Phys. Rev. Lett. 95, 226801 (2005).

3 B.A. Bernevig \& S.C. Zhang. Quantum spin Hall effect. Phys. Rev. Lett. 96, 106802 (2006).

4 B. A. Bernevig, T. L. Hughes \& S.C. Zhang. Quantum spin Hall effect and topological phase transition in $\mathrm{HgTe}$ quantum wells. Science 314, 1757 (2006).

${ }^{5}$ König, M. et al. Quantum spin Hall insulator state in $\mathrm{HgTe}$ quantum wells. Science 318, 766-770 (2007).

${ }^{6} \mathrm{Fu}, \mathrm{L}$. \& Kane, C. L. Topological insulators with inversion symmetry. Phys. Rev. B 76, 045302 (2007).

7 Moore, J. E. \& Balents, L. Topological invariants of time-reversal-invariant band structures. Phys. Rev. B $\mathbf{7 5}$, 121306 (2007).

8 Roy, R. On the $Z_{2}$ classification of quantum spin Hall models. arxiv: cond-mat/0604211

9 Qi, X.-L., Hughes, T. L. \& Zhang, S.-C. Topological field theory of time-reversal invariant insulators. Phys. Rev. B 78, 195424-43 (2008).

10 Hsieh, D. et al. A topological Dirac insulator in a quantum spin Hall phase. Nature 452, 970-974 (2008).

11 Zhang, S. C. The Chern-Simons-Landau-Ginzburg theory of the fractional quantum Hall effect. Int. J. Mod. Phys. B 6, 25 (1992).

12 Perdew, J. P., Burke, K. \& Ernzerhof, M. Generalized gradient approximation made simple. Phys. Rev. Lett. 77, 3865 (1996).

13 Hohenberg, P. \& Kohn, W. Inhomogeneous electron gas. Phys. Rev. 136, B864-B871 (1964).

14 Kohn, W. \& Sham, L. J. Self-consistent equations including exchange and correlation effects. Phys. Rev. 140, A1133-A1138 (1965).

15 Fang, Z. \& Terakura, K. Structural distortion and magnetism in transition metal oxides: crucial roles of orbital degrees of freedom. Journal of Physics: Condensed Matter 14, 3001-3014 (2002).
16 O. Madelung, U. R. \& Schulz, M. Non-Tetrahedrally Bonded Elements and Binary Compounds I (SpringerVerlag, Berlin, 1998).

17 S. K. Mishra, S. S. \& Jepsen, O. Electronic structure and thermoelectric properties of bismuth telluride and bismuth selenide. J. Phys: Condens. Matter 9, 461-470 (1997).

18 Larson, P. Effects of uniaxial and hydrostatic pressure on the valence band maximum in $\mathrm{Sb}_{2} \mathrm{Te}_{3}$ : an electronic structure study. Phys. Rev. B 74, 205113 (2006).

19 Black, J., Conwell, E. M., Seigle, L. \& Spencer, C. W. Electrical and optical properties of some M2-N3- semiconductors. J. Phys. Chem. Solids 2, $240-251$ (1957).

20 Mooser, E. \& Pearson, W. B. New semiconducting compounds. Phys. Rev. 101, 492-493 (1956).

21 Wittel, K. \& Manne, R. Atomic Spin-Orbit Interaction Parameters from Spectral Data for 19 Elements. Theoret. Chim. Acta (Berl.) 33, 347-349 (1974).

22 Marzari, N. \& Vanderbilt, D. Maximally localized generalized wannier functions for composite energy bands. Phys. Rev. B 56, 12847 (1997).

23 Souza, I., Marzari, N. \& Vanderbilt, D. Maximally localized wannier functions for entangled energy bands. Phys. Rev. B 65, 035109 (2001).

24 Sancho, M. P. L., Sancho, J. M. L. \& Rubio, J. Quick iterative scheme for the calculation of transfer matrices: application to Mo (100). Journal of Physics F: Metal Physics 14, 1205-1215 (1984).

25 Sancho, M. P. L., Sancho, J. M. L., Sancho, J. M. L. \& Rubio, J. Highly convergent schemes for the calculation of bulk and surface green functions. Journal of Physics F: Metal Physics 15, 851-858 (1985).

26 Winkler, R. Spin-Orbit Coupling Effects in TwoDimensional Electron and Hole Systems, vol. 191 of Springer Tracts in Modern Physics (Springer-Verlag, Berlin, 2003).

27 Koenig, M. et al. The quantum spin Hall effect: theory and experiment. J. Phys. Soc. Japan 77, 031007 (2008).

28 Dai, X., Hughes, T. L., Qi, X.-L., Fang, Z. \& Zhang, S.-C. Helical edge and surface states in $\mathrm{HgTe}$ quantum wells and 
bulk insulators. Physical Review B 77, 125319-6 (2008).

29 Noh, H.-J. et al. Spin-orbit interaction effect in the electronic structure of $\mathrm{Bi}_{2} \mathrm{Te}_{3}$ observed by angle-resolved photoemission spectroscopy. Europhys. Lett. 81, 57006 (2008).

${ }^{30}$ Urazhdin, S. et al. Surface effects in layered semiconduc- tors $\mathrm{Bi}_{2} \mathrm{Se}_{3}$ and $\mathrm{Bi}_{2} \mathrm{Te}_{3}$. Phys. Rev. B 69, 085313 (2004).

31 Qi, X.-L., Li, R.-D., Zang, J., \& Zhang, S.-C. Seeing the magnetic monopole through the mirror of topological surface states. arxiv: cond-mat/ 0811.1303 (2008). 